\title{
Features of the Magnetic Properties of Co/Si/Co Thin-Film Systems
}

\author{
E. E. Shalygina*, A. M. Kharlamova, A. A. Rozhnovskaya, \\ G. V. Kurlyandskaya, and A. V. Svalov \\ Department of Physics, Moscow State University, Moscow, 119991 Russia \\ Ural Federal University, Yekaterinburg, 620002 Russia \\ *e-mail: shal@magn.ru \\ Received June 27, 2013
}

\begin{abstract}
The magnetic properties of $\mathrm{Co} / \mathrm{Si} / \mathrm{Co}$ thin-film structures grown by magnetron sputtering have been studied using magnetooptical techniques. It is established that the saturation field $\left(H_{\mathrm{S}}\right)$ of trilayers exhibits oscillations as a function of the thickness of the semiconductor (silicon) interlayer. This behavior is explained by structural features of the $\mathrm{Co} / \mathrm{Si} / \mathrm{Co}$ system and the presence of antiferromagnetic exchange coupling between magnetic layers via the silicon interlayer.
\end{abstract}

DOI: $10.1134 / \mathrm{S} 1063785013120249$

The physical properties of thin magnetic films of $3 d$ transition metals and multilayer structures consisting of alternating submicron-thick magnetic and nonmagnetic layers are still among the most interesting objects for investigation in the physics of magnetic phenomena. The interest in these investigations is related, in particular, to the wide use of these systems in numerous devices of modern micro- and nanoelectronics based on the discovery of phenomena such as colossal magnetoresistance [1], antiferromagnetic exchange coupling between magnetic layers via nonmagnetic spacers [2], and oscillating exchange coupling between ferromagnetic layers $(\mathrm{Fe}, \mathrm{Co})$ via nonmagnetic metal $(\mathrm{Cu}, \mathrm{Ag}, \mathrm{Au}, \mathrm{Mo}, \mathrm{Ta}$, etc. ) spacers [3, 4]. Quantitative evaluation of the oscillating exchange coupling between magnetic layers in thin-film systems as dependent on the thickness of a nonmagnetic metal interlayer is usually based on the Ruderman-KittelKasuya-Yosida mechanism (RKKY interaction) and takes into account the quantum-confinement effects $[5,6]$, which are manifested by changes in the electron structure of ultrathin magnetic layers (the appearance of so-called quantum-well states) as compared to that of volume materials.

Despite considerable progress in investigation of the aforementioned materials, the physics of exchange interactions in thin-film magnetic structures with semiconductor spacers is far from complete understanding of the observed phenomena. The most interesting experimental investigations of the structural and magnetic properties have been reported for $\mathrm{Fe} / \mathrm{Si}$ [7-11] and $\mathrm{Co} / \mathrm{Si}[10-19]$ thin-film systems. Analysis of the available data showed that the antiferromagnetic exchange between iron layers in $\mathrm{Fe} / \mathrm{Si} / \mathrm{Fe}$ systems was observed for silicon layer thicknesses within 1.4-1.7 and 1.4-2.2 nm (see, e.g., [20, 21]). However, data on the exchange interaction between magnetic layers via nonmagnetic spacers in $\mathrm{Co} / \mathrm{Si} / \mathrm{Co}$ thin-film systems are rather contradictory. At the same time, it is known that magnetic transition metals $(\mathrm{Fe}, \mathrm{Co})$ are widely used in multilayer structures possessing colossal magnetoresistance, while silicon is a base element for modern micro- and nanoelectronics. Thus, the study of magnetic properties of systems consisting of magnetic metal layers with Si spacers is of special importance.

This Letter presents the results of an investigation of the properties of $\mathrm{Co} / \mathrm{Si} / \mathrm{Co}$ thin-film systems. In order to provide deeper insight into the behavior of $\mathrm{Co} / \mathrm{Si} / \mathrm{Co}$ trilayers in a magnetic field, we have thoroughly studied the influence of the thickness of silicon interlayer on these magnetic characteristics of samples.

The experiments were performed with $\mathrm{Co} / \mathrm{Si} / \mathrm{Co}$ three-layer structures grown by sequential magnetron sputter deposition of Co and Si layers onto glass substrates at room temperature. The samples were prepared in a vacuum chamber with a residual pressure of $3.8 \times 10^{-7}$ mbar. The working gas (argon) pressure during sputtering was on the order of $3.8 \times 10^{-3}$ mbar. The thickness of cobalt layers in all samples was $t_{\mathrm{Co}}=5 \mathrm{~nm}$. The thickness of the silicon interlayer was varied within $t_{\mathrm{Si}}=0.2-3.2 \mathrm{~nm}$.

The structure of thin-film systems was studied by $\mathrm{X}$-ray diffraction (XRD) using $\mathrm{Cu} K_{\alpha}$ radiation. The presence of periodic structures with clear interfaces between layers was confirmed by data of small-angle $\mathrm{X}$-ray scattering (SAXS). The thicknesses of individual layers were determined by the deposition rate and time and checked by the SAXS data. The surface morphol- 


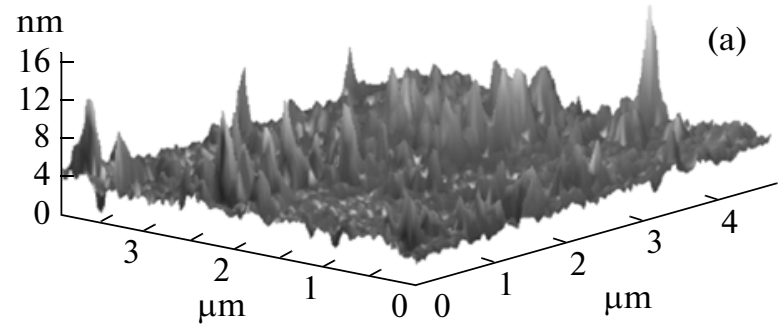

(b)

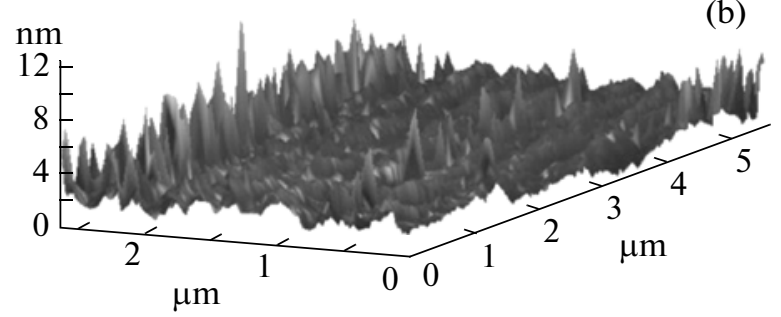

Fig. 1. Typical AFM images of (a) Co/0.2 nm Si/Co and (b) $\mathrm{Co} / 3 \mathrm{~nm} / \mathrm{Si} / \mathrm{Co}$ trilayers.

ogy of samples was studied by atomic force microscopy (AFM).

The magnetic characteristics of $\mathrm{Co} / \mathrm{Si} / \mathrm{Co}$ trilayers were determined on a magnetooptical magnetometer [22] using the equatorial Kerr effect. The hysteresis loops were measured for two orientations of external magnetic field $H$ in the plane of the structure, whereby the field was either parallel (D1 direction) or perpendicular (D2 direction) to the direction of the field $\left(H_{\text {sub }}=250 \mathrm{Oe}\right)$ that was applied (parallel to the substrate surface) during the deposition of films. All measurements were performed in air at room temperature.

The results of XRD measurements showed that cobalt layers in the samples studied have a nanocrystalline structure with grain dimensions (calculated by the Scherrer method) on the order of layer thickness. Figure 1 shows characteristic results of the AFM examination of $\mathrm{Co} / \mathrm{Si} / \mathrm{Co}$ trilayers. Analysis of these data using a special program (developed for the particular instrument employed in this work) showed that the sample surface roughness is on the order of $R_{a}=$ $0.4-0.6 \mathrm{~nm}$, where $R_{a}$ is independent of the thickness of a silicon layer. This experimental fact indicates that, as the silicon interlayer thickness is varied, the surface quality of samples must not influence their behavior in the magnetic field.

Figure 2 shows typical hysteresis loops observed in a magnetic field applied parallel to the D1 or D2 directions. Results of these measurements were used to study saturation field $H_{\mathrm{S}}$ of $\mathrm{Co} / \mathrm{Si} / \mathrm{Co}$ trilayers as a function of silicon-layer thickness $t_{\mathrm{Si}}$ (see Fig. 3).

Analysis of the results of magnetic measurements showed that hysteresis loops measured in magnetic fields applied in the D1 and D2 directions are significantly different (Fig. 2), which is indicative of the existence of induced planar magnetic anisotropy. According to the existing model [23], this anisotropy is
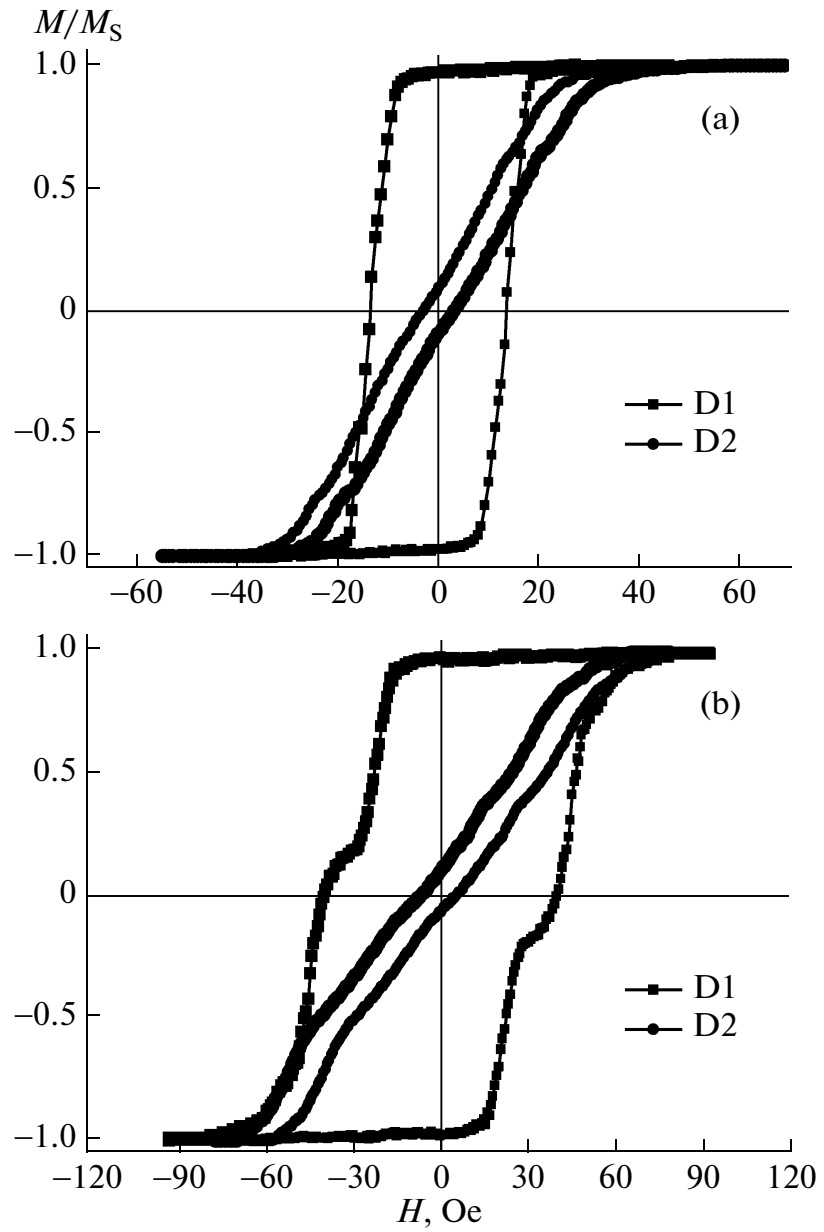

Fig. 2. Hysteresis loops observed for $\mathrm{Co} / \mathrm{Si} / \mathrm{Co}$ trilayers with $t_{\mathrm{Si}}=1.2 \mathrm{~nm}$ (a) and $2.2 \mathrm{~nm}$ (b) in a magnetic field parallel to the D1 or D2 direction.

induced by field $H_{\text {sub }}$ that was applied during the deposition of thin-film systems. At present, the main mechanism responsible for the appearance of induced magnetic anisotropy is commonly considered to be related to a pairwise ordering of atoms [23]. In the case of D2 direction, the hysteresis loops are inclined and characterized by rather low values of the coercive field and remanent magnetization. This behavior of magnetic trilayers is observed for their magnetization reversal along the hard axis, which takes place predominantly due to rotation of the magnetization vector. The shape of hysteresis loops measured in the magnetic field applied in the D1 direction (i.e., parallel to the easy axis) depends on the silicon layer thickness. In particular, it was found that the samples with $t_{\mathrm{Si}}<1.6 \mathrm{~nm}$ exhibited almost rectangular loops (see Fig. 2a), while the samples with $t_{\mathrm{Si}}>1.6 \mathrm{~nm}$ exhibited two-step loops (Fig. 2b). Saturation field $\left(H_{\mathrm{S}}\right)$ of $\mathrm{Co} / \mathrm{Si} / \mathrm{Co}$ trilayers also depends on the silicon-layer thickness and, as can be seen from Fig. $3, H_{\mathrm{S}}$ oscillates with increasing $t_{\mathrm{Si}}$. 
The obtained data can be explained as follows. As commonly represented (see, e.g., [4]), all samples with a rectangular hysteresis loop have parallel orientation of magnetization in adjacent metal layers. The magnetization reversal in these systems is analogous to the process in single-layer thin-film samples and can proceed, e.g., via irreversible growth of the remagnetization nuclei. The variation of $H_{\mathrm{S}}$ with increasing thickness of the silicon interlayer can be explained based on the following facts. As is known [9], the entire interlayer in the case of very small $t_{\mathrm{Si}}$ consists of cobalt silicide. As $t_{\mathrm{Si}}$ increases, an additional layer of silicon appears near the cobalt-silicide layer at $\mathrm{Co} / \mathrm{Si}$ interfaces, which leads to an initial increase in $H_{\mathrm{s}}$. In addition, the behavior of trilayers in magnetic fields is influenced by stray fields created by cobalt layers, which decrease the effective applied magnetic field acting on the sample. However, the further increase in the silicon-spacer thickness decreases the magnetostatic interaction between cobalt layers. Thus, the aforementioned features of the $\mathrm{Co} / \mathrm{Si} / \mathrm{Co}$ system determine the observed behavior of $H_{\mathrm{S}}\left(t_{\mathrm{Si}}\right)$ for $t_{\mathrm{Si}}<$ $1.6 \mathrm{~nm}$.

According to our recent experimental data [4, 24] and results of model calculations [25], the two-step hysteresis loops observed for $1.6 \mathrm{~nm}<t_{\mathrm{Si}}<3.2 \mathrm{~nm}$ are indicative of the antiparallel orientation of magnetization in cobalt layers and, hence of the antiferromagnetic exchange between ferromagnetic layers via the silicon spacer. By analogy with the case of $\mathrm{Fe} / \mathrm{Mo}$ and $\mathrm{Ta} / \mathrm{Fe}$ thin-film systems $[4,24]$, the observed $H_{\mathrm{S}}\left(t_{\mathrm{Si}}\right)$ dependence can be explained by variation of the constant $J_{\mathrm{AF}}$ of antiferromagnetic exchange between cobalt layers with increasing $t_{\mathrm{Si}}$. Estimation of the maximum value of this parameter $J_{\mathrm{AF}}^{\mathrm{max}}$ from the wellknown formula $J_{\mathrm{AF}} \approx H_{\mathrm{S}} M_{\mathrm{S}} t_{\mathrm{Co}} / 4$ gives $J_{\mathrm{AF}}^{\max } \approx$ $0.016 \mathrm{erg} / \mathrm{cm}^{2}$, which is almost tenfold smaller than the values observed in Fe/Si thin-film structures [21]. As for the mechanism of exchange coupling between magnetic layers in the presence of a semiconductor spacer, it differs from the case of a metallic interlayer. According to the existing notions [20,21], a possible mechanism of this exchange in $\mathrm{Fe}, \mathrm{Co} / \mathrm{Si} / \mathrm{Fe}$, Co thinfilm systems is related to the tunneling of polarized electrons via localized defect states which are always present in the semiconductor layer.

In conclusion, we have studied the magnetic properties of $\mathrm{Co} / \mathrm{Si} / \mathrm{Co}$ thin-film structures. It is established that the shape of hysteresis loops of these trilayers depends on thickness $t_{\mathrm{Si}}$ of the silicon interlayer. Two-step hysteresis loops, which have been observed for $1.6 \mathrm{~nm}<t_{\mathrm{Si}}<3.2 \mathrm{~nm}$, are explained by the antiferromagnetic exchange coupling between cobalt layers via the semiconductor silicon spacer and the oscillatory character of this interaction. The obtained experimental data should be taken into account in develop-

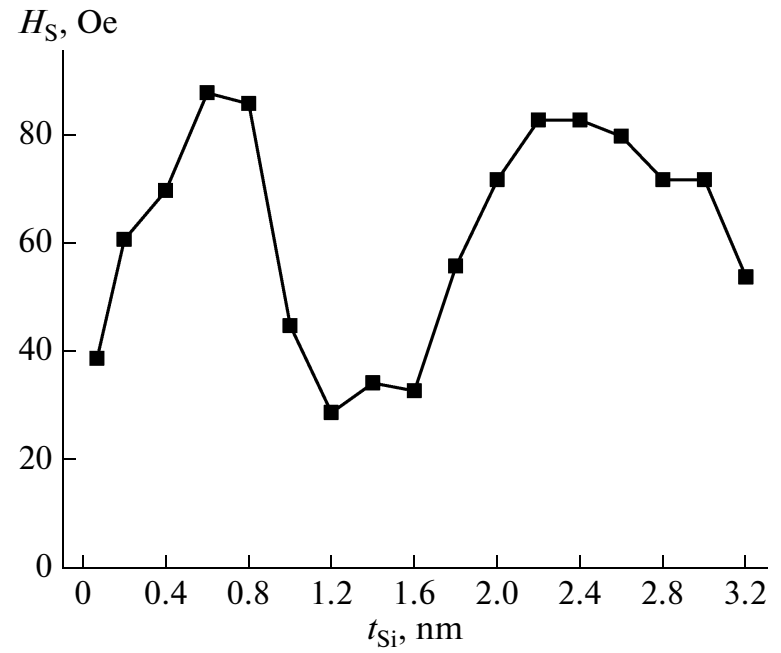

Fig. 3. Plots of saturation field $H_{\mathrm{S}}$ vs. silicon-spacer thickness $t_{\mathrm{Si}}$ for $\mathrm{Co} / \mathrm{Si} / \mathrm{Co}$ thin-film system.

ing nanostructural layered magnetic materials for modern microelectronic devices.

\section{REFERENCES}

1. N. Babich, J. M. Broto, A. Fert, F. Nguyen Van Dau, and F. Petroff, Phys. Rev. Lett. 61, 2472 (1988).

2. S. P. Parkin, N. More, and K. P. Roche, Phys. Rev. Lett. 64, 2304 (1990).

3. P. Grunberg, R. Schreiber, Y. Pang, M. B. Brodsky, et al., Phys. Rev. Lett. 57, 2442 (1986).

4. E. E. Shalygina, A. A. Rojnovskaya, and A. N. Shalygin, Solid State Phenom. 190, 514 (2012).

5. M. D. Stiles, Phys. Rev. 48, 7238 (1993).

6. P. Bruno, Phys. Rev. B 52, 411 (1995).

7. A. Paul, M. Buchmeir, D. E. Burger, U. Rucker, and C. M. Schneider, Phys. Rev. B 77, 184409 (2008).

8. S. R. Naik, S. Rai, M. K. Chattopahyay, et al., J. Appl. Phys. 104, 063525 (2008).

9. S. R. Naik, S. Rai, M. K. Tiwari, and G. S. Lodha, J. Phys. D: Appl. Phys. 41, 115307 (2008).

10. T. Lucinski, M. Kopcewicz, A. Hutten, et al., Mater. Sci. 21, 25 (2003).

11. T. Lucinski, P. Wandziuk, F. Stobieski, et al., J. Magn. Magn. Mater. 282, 248 (2004).

12. P. J. Grundy, J. M. Fallon, and H. J. Blythe, Phys. Rev. B 62, 9566 (2000).

13. J. M. Fallon, C. A. Faunce, and P. J. Grundy, J. Appl. Phys. 88, 2400 (2000).

14. P. Ruterana, P. Haudy, and P. Boher, J. Appl. Phys. 68, 1033 (1990).

15. J. Enkovaara, A. Ayuela, and R. M. Nieminen, Phys. Rev. B 62, 16018 (2000).

16. J. S. Tsay, Y. D. Yao, Y. Liou, S. F. Lee, and C. S. Yang, J. Magn. Magn. Mater. 209, 208 (2000).

17. J. S. Tsay, T. Y. Eu, M. H. Lin, C. S. Yang, and Y. D. Yao, Appl. Phys. Lett. 88, 102506 (2006). 
18. A. Kharmouche, S.-M. Cherif, A. Bourzami, A. Layadi, and G. Schmerber, J. Phys. D: Appl. Phys. 37, 2583 (2004).

19. A. Sagdeo, S. Rai, A. K. Srivastava, G. S. Lodha, R. Rawat, K. Le Guen, and P. Jonnard, J. Phys.: Condens. Mater. 23, 246004 (2011).

20. B. Briner and M. Landolt, Phys. Rev. Lett. 73, 340 (1994).

21. J. E. Mattson, Fric E. Fillerton, S. Kumar, S. R. Lee, et al., J. Appl. Phys. 10, 6169 (1994).
22. E. E. Shalyguina, M. A. Komarova, and V. V. Molokanov, J. Exp. Theor. Phys. 95, 511 (2002).

23. S. Chikazumi, Physics of Magnetism (John Willey \& Sons, New York, 2001), p. 554.

24. E. E. Shalygina, A. A. Rozhnovskaya, and A. N. Shalygin, Tech. Phys. Lett. 37, 980 (2011).

25. A. K. Zvezdin, Phys. Solid State 42, 120 (2000).

Translated by P. Pozdeev 